ARTICLE

DOI: $10.1038 / \mathrm{s} 41467-018-06850-6$

\title{
Continuous negative-to-positive tuning of thermal expansion achieved by controlled gas sorption in porous coordination frameworks
}

Josie E. Auckett', Arnold A. Barkhordarian², Stephen H. Ogilvie'2,

Samuel G. Duyker (D) ${ }^{2}$, Hubert Chevreau', Vanessa K. Peterson (D) ${ }^{1} \&$ Cameron J. Kepert (D) ${ }^{2}$

Control of the thermomechanical properties of functional materials is of great fundamental and technological significance, with the achievement of zero or negative thermal expansion behavior being a key goal for various applications. A dynamic, reversible mode of control is demonstrated for the first time in two Prussian blue derivative frameworks whose coefficients of thermal expansion are tuned continuously from negative to positive values by varying the concentration of adsorbed $\mathrm{CO}_{2}$. A simple empirical model that captures site-specific guest contributions to the framework expansion is derived, and displays excellent agreement with the observed lattice behaviour.

\footnotetext{
${ }^{1}$ Australian Centre for Neutron Scattering, Australian Nuclear Science and Technology Organisation, Lucas Heights, NSW 2234, Australia. ${ }^{2}$ School of Chemistry, The University of Sydney, Sydney, NSW 2006, Australia. These authors contributed equally: Josie E. Auckett, Arnold A. Barkhordarian, Stephen H. Ogilvie. Correspondence and requests for materials should be addressed to S.G.D. (email: samuel.duyker@sydney.edu.au) or to V.K.P. (email: vanessa.peterson@ansto.gov.au) or to C.J.K. (email: cameron.kepert@sydney.edu.au)
} 
C oordination frameworks have attracted sustained research interest in recent years due to their various properties of potential industrial and technological relevance ${ }^{1,2}$. Among the most notable of these is an ability to selectively adsorb and release small-molecule guests over many operating cycles $^{3-6}$. This functionality is critical to the ongoing quest for efficient gas storage and separation materials in numerous industrial contexts, such as post-combustion carbon dioxide capture and hydrogen fuel storage $\mathrm{e}^{7-9}$. The inherent porosity and relative structural flexibility that facilitate guest sorption in many frameworks also contribute to the remarkable thermomechanical behaviours exhibited by some of these materials; ${ }^{10,11}$ for example, dynamic motions of framework structural components into the available void spaces give rise to negative thermal expansion (NTE) in a number of frameworks ${ }^{12-16}$. NTE and zero thermal expansion (ZTE) are considered desirable material properties for a variety of applications where the minimisation of strain during temperature cycling is important, and the ability to tune the rate of expansion of a device component to compensate for the positive expansion of another component is particularly advantageous. As the inclusion of guests or other modifiers in the voids of porous frameworks can directly influence the dynamic motions associated with NTE, the interplay between guest concentration and temperature introduces considerable complexity to the study of thermomechanical properties in host-guest framework systems ${ }^{17-19}$.

The Prussian blue family $\left(M_{y}\left[M^{\prime}(\mathrm{CN})_{6}\right], M\right.$ and $M^{\prime}=$ transition metals) comprises one of the simplest coordination framework structure types and is a model system often suited to the study of phenomena common to more complicated systems. The archetypical Prussian blue framework is constructed of cyanido $\left(\mathrm{CN}^{-}\right)$ligands bridging the octahedrally coordinated $M$ and $M^{\prime}$ ions, which are arranged in a "rock salt" ordering pattern on a cubic lattice. $M$ and $M^{\prime}$ may be chosen with various combinations of valence states ${ }^{20-24}$, generating a series of structurally analogous frameworks known as Prussian blue analogues (PBAs). Many of the possible valence combinations result in well-defined concentrations of randomly distributed hexacyanidometallate vacancies in order to preserve charge balance (Fig. 1). The faces of the cubic "large pore" created by an absent hexacyanidometallate unit each contain a bare metal site that can act as an attractive site for guest binding ${ }^{20,25}$. An important PBA variant is the transition metal nitroprusside (TMNP) family ${ }^{26}$, in which $\left[\mathrm{Fe}(\mathrm{CN})_{5} \mathrm{NO}\right]$ is substituted for $\left[M^{\prime}(\mathrm{CN})_{6}\right]$ and additional "metal vacancy pores" are introduced adjacent to every six non-bridging NO ligands.

Various PBA and derivative frameworks have been shown to exhibit isotropic NTE over a wide temperature range due to a mechanism in which transverse ligand vibrations into the adjacent void spaces cause the metal-centred octahedral units to be drawn closer together ${ }^{17,21}$. Previous studies have demonstrated that the coefficient of thermal expansion (CTE, $\alpha$ ) of PBA and other NTE-active frameworks can be varied considerably, even from negative to positive values, by the substitution of different metal ions ${ }^{21}, 27,28$. It has also been shown that NTE behaviour in certain frameworks is converted to positive thermal expansion when solvent molecules are present in the pores ${ }^{17,29,30}$ and that intermediate CTE values can be achieved by partial desolvation at high temperatures ${ }^{18}$. Substitution of different solvent guests has also been shown to modify CTEs in uniaxial and biaxial NTE materials ${ }^{31,32}$. The presence of extra-framework ionic species has additionally been shown to influence thermal expansion behaviour $^{33}, 34$. However, to our knowledge, no studies have yet shown how the CTE of one framework can be tuned continuously and reversibly over a wide range of positive and negative values using a dynamic stimulus. Here we demonstrate a new and facile method of negative-to-positive CTE tuning by altering the concentration of $\mathrm{CO}_{2}$ guest molecules adsorbed in the pores, as
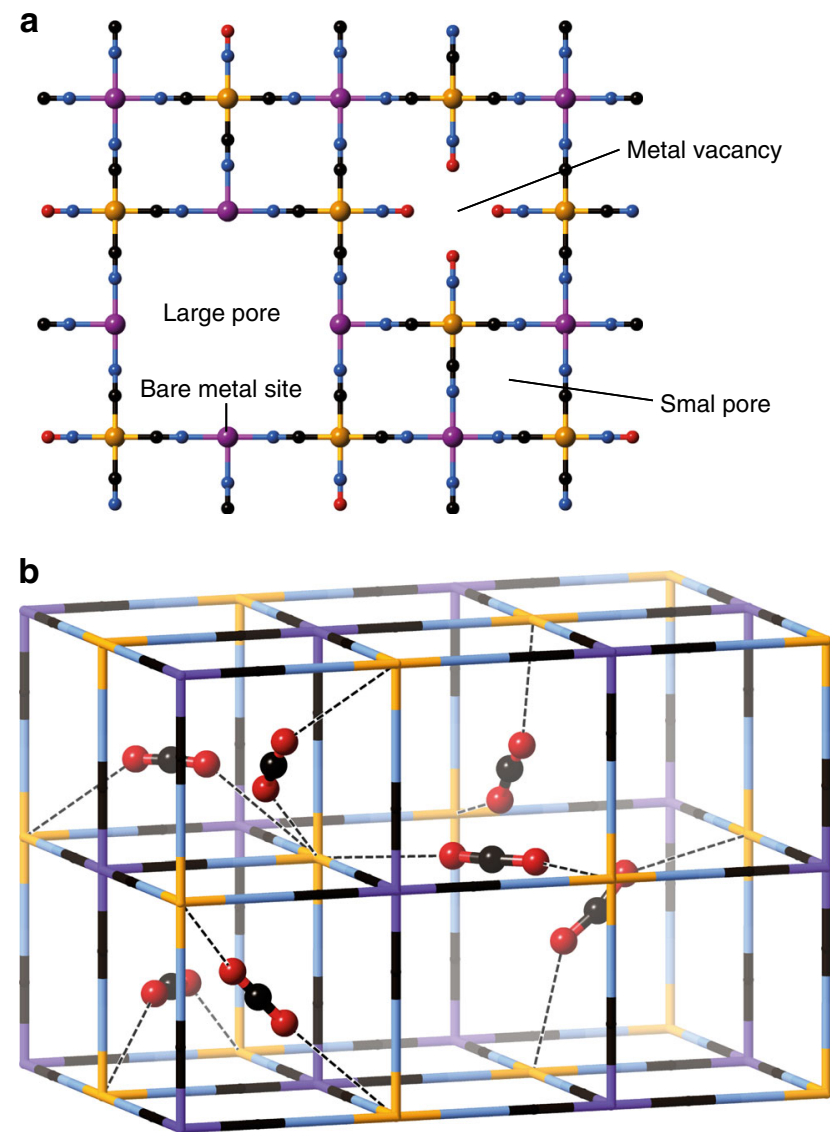

Fig. 1 Visual representations of Prussian blue-type frameworks. a Network of a defective TMNP framework illustrating the different pore types. The large pore arises from vacancies of the $\mathrm{Fe}(\mathrm{CN})_{5} \mathrm{NO}$ unit in TMNPs or of the $\mathrm{M}^{\prime}(\mathrm{CN})_{6}$ unit in PBAs. The metal vacancy pore arises from the presence of non-bridging NO ligands in TMNPs and does not occur in

hexacyanidometallate PBAs. Note that, while they are shown as separate here, the vacancy pores may border upon or overlap with others of the same or different type. $\mathbf{b}$ Fragment of the FeCo-PB structure containing its theoretical maximum $\mathrm{CO}_{2}$ content of one "Site $A$ " $\mathrm{CO}_{2}$ molecule in each of

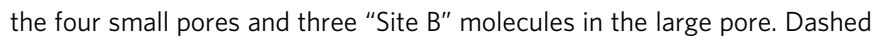
lines indicate interactions between $\mathrm{CO}_{2}$ and the Fe sites. The host and guest atomic positions were previously determined by NPD ${ }^{37}$. Colours: Fe (orange); Co (purple); C (black); N (blue); O (red)

exhibited by the TMNP $\mathrm{Co}\left[\mathrm{Fe}(\mathrm{CN})_{5} \mathrm{NO}\right]$ (hereafter Co-NP) and the PBA $\mathrm{Fe}_{3}\left[\mathrm{Co}(\mathrm{CN})_{6}\right]_{2}$ (hereafter FeCo-PB). A simple empirical model for the modifying effects of guest concentration on the thermal expansion characteristics of the empty framework is also developed, allowing the observed behaviour to be understood in relation to the guest-binding locations and host-guest interactions within the host frameworks.

\section{Results}

Tuneable guest-dependent thermal expansion. The unit cell parameters of Co-NP and $\mathrm{FeCo}-\mathrm{PB}$ at various $\mathrm{CO}_{2}$ loadings were extracted by Le Bail analysis of variable-temperature neutron powder diffraction (NPD) data collected upon warming (Fig. 2a, b; experiment details in ESI). The sharp downturn observed at high temperatures for the highest $\mathrm{CO}_{2}$ concentrations is attributed to the onset of thermally induced $\mathrm{CO}_{2}$ desorption, which causes the lattice parameter to approach that of the guest-free framework. For both FeCo-PB and Co-NP, linear CTEs $\left(\alpha_{a}=(1 /\right.$ $a)(\mathrm{d} a / \mathrm{d} T))$ calculated from linear regression fits in the low- 

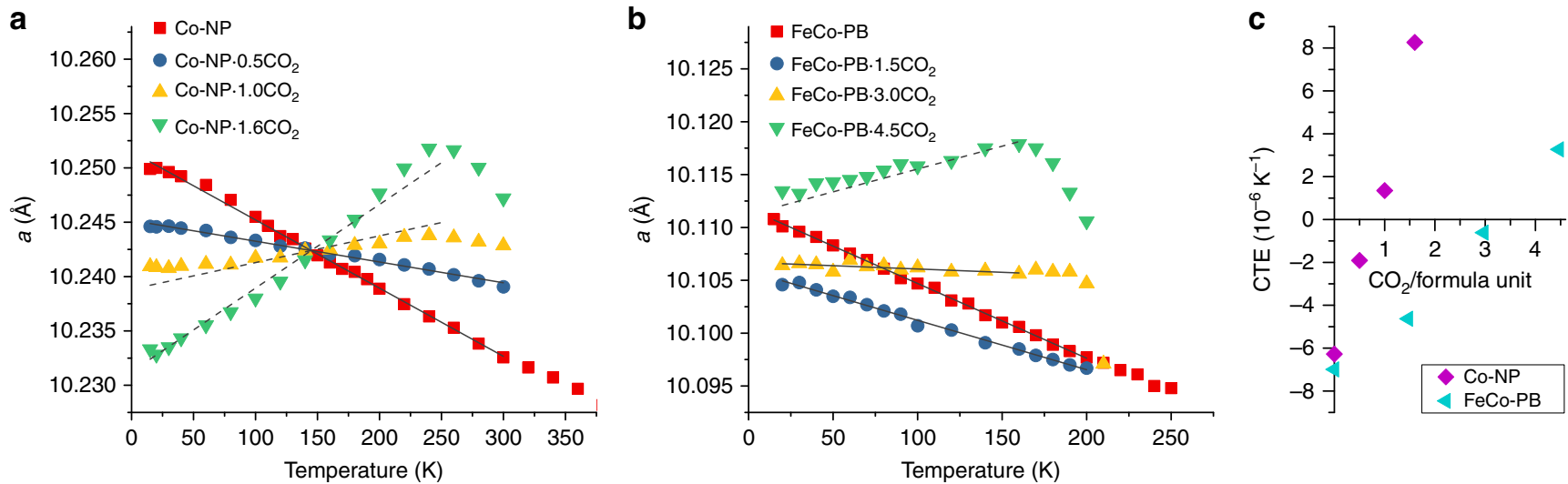

Fig. $2 \mathrm{CO}_{2}$-dependent thermal expansion trends for Co-NP and FeCo-PB. a, b Symbols represent the experimentally determined cubic lattice parameters of $\mathrm{Co}-\mathrm{NP}$ and $\mathrm{FeCo}-\mathrm{PB}$ at various $\mathrm{CO}_{2}$ loadings and temperatures. Error bars are smaller than the symbols. Solid lines represent linear regression fits over limited data ranges (see Table 1); these fits were used to parameterise the formulae derived in the text (see Eqs. (1) and (2)). The dashed trend lines are predicted by the parameterised model. c Evolution of the CTE values obtained by linear regression fits against all data series

\begin{tabular}{|c|c|c|c|c|}
\hline \multicolumn{3}{|c|}{$\begin{array}{l}x\left(\mathrm{CO}_{2} \text { guests per formula }\right. \\
\text { unit) }\end{array}$} & $\alpha_{a}\left(\times 10^{-6} K^{-1}\right)$ & Fitted range (K) \\
\hline \multicolumn{5}{|l|}{$\overline{C o-N P}$} \\
\hline 0 & & & $-6.12(8)$ & $15-300$ \\
\hline 0.5 & & & $-1.87(5)$ & $15-300$ \\
\hline 1.0 & & & $+1.32(8)$ & $15-240$ \\
\hline 1.6 & & & $+8.1(3)$ & $15-240$ \\
\hline $\mathrm{FeCo}-\mathrm{PB}$ & Site $A$ & Site $B$ & & \\
\hline 0 & 0 & 0 & $-6.99(6)$ & $15-200$ \\
\hline 1.5 & 1.5 & 0 & $-4.63(10)$ & $20-200$ \\
\hline 3.0 & 2.0 & 1.0 & $-0.6(2)$ & $20-160$ \\
\hline 4.5 & 2.0 & 2.5 & $+3.30(17)$ & $20-160$ \\
\hline
\end{tabular}

temperature range display a continuous increase with increasing $\mathrm{CO}_{2}$ concentration, from strongly negative for the empty framework to positive for the highest $\mathrm{CO}_{2}$ concentrations measured (Table 1). The values obtained for the empty frameworks are typical of guest-free PBAs ${ }^{21}$.

The tunability of thermal expansion in these frameworks is rationalised by considering the nature of low-energy, rigid-unit NTE modes operating in PBAs and their derivatives ${ }^{13}, 14$. As the empty pore space of the framework becomes filled with guests, the transverse vibrations of the bridging cyanides, which ordinarily lead to concerted, volume-reducing distortions of the framework, are increasingly impeded by steric interactions with the guest molecules. This explanation has also been proposed for the suppression of NTE by the presence of solvent molecules in the $M\left[\mathrm{Pt}(\mathrm{CN})_{6}\right](M=\mathrm{Zn}, \mathrm{Cd})^{17}$ and $\mathrm{Cd}(\mathrm{CN})_{2}{ }^{18}$ frameworks.

Simple expansivity model. In Co-NP, the intersection of all lattice parameter trends at a single point is suggestive of competition between two linear factors of opposite sign affecting thermal expansion simultaneously and negating each other at a certain temperature. We assign these competing effects to two different interactions known to occur between guest molecules and porous frameworks. The first has been reported in zeolites ${ }^{35}$ and MOFs ${ }^{36}$ and is characterised by contraction of the framework at low temperatures due to the attractive nature of the guest acting on multiple framework atoms; this may be termed the "static" effect. Simultaneously, the guests exert an expansive pressure on the framework with increasing thermal
Table 2 Parameter values determined for the prediction of guest-dependent thermal expansion trends in Co-NP and FeCo-PB

Co-NP (using Eq. (1))

\begin{tabular}{llll}
\hline$a_{0}$ & $10.25150(13) \AA$ & $a_{0}$ & $10.11177(7) \AA$ \\
$m_{0}$ & $-6.28(8) \times 10^{-5} \AA K^{-1}$ & $m_{0}$ & $-7.07(6) \times 10^{-5} \AA K^{-1}$ \\
$S$ & $-12.7(3) \times 10^{-3} \AA$ & $S_{A}$ & $-4.00(9) \times 10^{-3} \AA$ \\
$D$ & $8.74(19) \times 10^{-5} \AA K^{-1}$ & $D_{A}$ & $1.63(8) \times 10^{-5} \AA K^{-1}$ \\
& & $S_{B}$ & $3.0(3) \times 10^{-3} \AA$ \\
& & $D_{B}$ & $3.3(3) \times 10^{-5} \AA K^{-1}$ \\
\hline \multicolumn{2}{l}{} \\
\multicolumn{2}{l}{$a_{0}$ and $m_{0}$ originate from linear regression fits to the empty framework data } \\
\hline
\end{tabular}

excitation, which may be called the "dynamic" or "thermal" effect. Contraction of the framework due to the static effect of $\mathrm{CO}_{2}$ is considered to be temperature independent and has the effect of decreasing the $0 \mathrm{~K}$ intercept of the thermal expansion trend with increasing guest concentration. By contrast, the temperaturedependent dynamic effect positively increases the rate of thermal expansion with increasing guest concentration. The following simple expression captures the static and dynamic effects on thermal expansion behaviour:

$$
a=a_{0}+S x+\left(m_{0}+D x\right) T
$$

where $x$ is the guest concentration, $T$ is temperature, $a_{0}$ is the extrapolated lattice parameter of the empty framework at $0 \mathrm{~K}, m_{0}$ is the thermal expansion gradient of the empty framework, $S$ is the static parameter or contractive guest effect $(\AA)$, and $D$ is the dynamic thermal parameter or expansive guest effect $\left(\AA \mathrm{K}^{-1}\right)$. It may be seen that when $D T=-S$, the concentration-dependent expansive and contractive effects are cancelled out; consequently, thermal expansion trends for all guest concentrations intersect at $T=-S / D$. Furthermore, zero thermal expansion (ZTE) behaviour is predicted when the guest concentration $x=-m_{0} / D$. In the case of Co-NP, the calculated crossover temperature for all curves is $145 \mathrm{~K}$ and ZTE is predicted to occur at a guest concentration of $0.72(2) \mathrm{CO}_{2} /$ formula unit, with the associated unit cell parameter $a=10.2424(3) \AA$. The values determined for $S$ and $D$ are reported in Table 2; details of their calculation are given in Supplementary Information. Thermal expansion trends predicted by the model are represented with dashed lines in Fig. 2a, revealing excellent agreement with the experimental data below the onset temperature of $\mathrm{CO}_{2}$ desorption. 
Multi-site expansivity model. The wider applicability of this approach for modelling thermal expansion in cubic systems is expected to be dependent upon the nature of guest-binding sites within the host framework. Indeed, attempts to model the thermal expansion behaviour of FeCo-PB using Eq. (1) were not successful. Ogilvie et al. ${ }^{37}$ previously identified two distinct "bridged" binding sites for $\mathrm{CO}_{2}$ in $\mathrm{FeCo}-\mathrm{PB}$ at $15 \mathrm{~K}$ : Site $\mathrm{A}$ in the large pore (maximum capacity $2 \mathrm{CO}_{2}$ molecules per formula unit), and Site B located over the small-pore squares. A modified equation taking into account different static and dynamic contributions of guests bound at Sites A and B takes the form

$$
a=a_{0}+S_{A} x_{A}+S_{B} x_{B}+\left(m_{0}+D_{A} x_{A}+D_{B} x_{B}\right) T
$$

where the subscripts $A$ and $B$ denote guest concentrations and thermal expansion parameters corresponding to $\mathrm{CO}_{2}$-binding sites $\mathrm{A}$ and $\mathrm{B}$, respectively. Assuming maximal occupation of Site A followed by Site B up to the nominal guest concentration (Table 1), a surprisingly good approximation of the experimental data at $4.5 \mathrm{CO}_{2}$ is obtained using the extended model in Eq. (2), lending support to the suitability and predictive power of this model for multisite sorbents. The fact that the model succeeds by assuming perfectly sequential filling of Sites $\mathrm{A}$ and $\mathrm{B}$ also suggests that thermal randomisation of $\mathrm{CO}_{2}$ guests over both sites is negligible for all measured $\mathrm{CO}_{2}$ concentrations up to the desorption onset temperature; this is consistent with the highly optimal binding geometry identified for Site $\mathrm{A}^{37}$, which is expected to give it a significant energetic advantage over Site B.

The $D_{A}$ and $D_{B}$ values in Table 2 indicate that $\mathrm{CO}_{2}$ guests bound at Site $\mathrm{B}$ in $\mathrm{FeCo}-\mathrm{PB}$ cause a more rapid positive increase of the CTE than those at Site A. Given that Site B is positioned closer than Site A to the $\mathrm{CN}^{-}$ligands ${ }^{37}$, this observation is consistent with the suppression of NTE being caused by interference of $\mathrm{CO}_{2}$ guests with the $\mathrm{CN}^{-}$vibrational modes. Guests at Site $\mathrm{B}$ are also found to exert an expansive static effect on the FeCo-PB framework $\left(S_{B}>0\right)$, probably reflecting the weaker host-guest interaction at $\mathrm{B}$ that is associated with the strong guest preference for Site A. By contrast, the fact that Co-NP was satisfactorily modelled using the simpler Eq. (1) suggests that it contains only one unique $\mathrm{CO}_{2}$-binding site or multiple sites of very similar character. This prediction is borne out by the preliminary results of Rietveld refinements against the NPD data for Co-NP, which have only identified a single, highly disordered $\mathrm{CO}_{2}$-binding site located at the centre of the small pore (see Supplementary Fig. 2). A detailed analysis of these refinements will be presented in a future publication.

Summary. Variable-temperature neutron diffraction data reveal for the first time that the CTEs of cyanide-based frameworks can be varied from negative to positive values by progressive adsorption of guest molecules into the pores. The evolution of CTE with guest concentration can be modelled on the basis of two independent contributions from each guest-binding site, as demonstrated by applying one-site and two-site versions of the model to Co-NP and FeCo-PB, respectively; this result further highlights the value of identifying and characterising individual guestbinding sites and understanding host-guest interactions in sorbent systems. The generally linear relationship between CTE and $\mathrm{CO}_{2}$ concentration offers a method of control for the tuning of thermal expansion properties in porous solids that is more facile than framework compositional changes or ex situ removal of solvent molecules, allowing the target concentration for generating ZTE behaviour to be calculated easily from the model equations and conveniently achieved through controlled gas dosing. This finding opens new opportunities for tailored thermal expansion properties to be dynamically engineered in porous framework materials over low-to-moderate temperature ranges.

\section{Methods}

Synthesis of $\mathbf{F e}_{3}\left[\mathbf{C o}(\mathbf{C N})_{6}\right]_{\mathbf{2}}$ (FeCo-PB). Aqueous solutions of $\mathrm{FeCl}_{2} \cdot \mathrm{H}_{2} \mathrm{O}(2.13 \mathrm{~g}$, $10.7 \mathrm{mmol})$ and $\mathrm{K}_{3} \mathrm{Co}(\mathrm{CN})_{6}(1.69 \mathrm{~g}, 5.08 \mathrm{mmol})$ were mixed dropwise with vigorous stirring. The solution was allowed to stand for $24 \mathrm{~h}$ before the precipitate was filtered and washed with water $(100 \mathrm{~mL})$, followed by ethanol $(100 \mathrm{~mL})$, and dried in air to yield $\mathrm{Fe}_{3}\left[\mathrm{Co}(\mathrm{CN})_{6}\right]_{2} \cdot x \mathrm{H}_{2} \mathrm{O}$ as a pale yellow powder (1.76 g; $x \approx 10$; yield $\sim 89 \%)$. Desolvation was achieved by grinding the sample to a fine powder and heating it in a glass sample tube at $423 \mathrm{~K}$ under high vacuum $\left(\sim 10^{-5} \mathrm{mbar}\right)$.

Synthesis of $\mathrm{Co}\left[\mathrm{Fe}(\mathrm{CN})_{5} \mathrm{NO}\right] \cdot 4 \mathrm{H}_{2} \mathrm{O}$ (Co-NP). A solution of $\mathrm{Na}_{2}\left[\mathrm{Fe}^{\mathrm{II}}\right.$ $\left.(\mathrm{CN})_{5} \mathrm{NO}\right] \cdot 2 \mathrm{H}_{2} \mathrm{O}(1.000 \mathrm{~g}, 3.36 \mathrm{mmol})$ in $200 \mathrm{~mL}$ deionised water was stirred until fully dissolved, followed by addition of $\mathrm{Co}\left(\mathrm{NO}_{3}\right)_{2} \cdot 6 \mathrm{H}_{2} \mathrm{O}(0.978 \mathrm{~g}, 3.36 \mathrm{mmol})$ to the stirred solution. After continuously stirring for $16 \mathrm{~h}$, the reaction mixture was centrifuged at $4000 \mathrm{rpm}$ and the supernatant discarded. The product was washed four times by adding fresh deionised water, agitating by hand and then repeating the centrifuge procedure. The resulting solid was air dried for $48 \mathrm{~h}$ and then stored in a desiccator for $24 \mathrm{~h}$. The product was a pale reddish brown powder (yield: $\sim 70 \%$ ).

Neutron powder diffraction. Variable-temperature NPD data were collected for Co-NP using the high-intensity neutron powder diffractometer WOMBAT ${ }^{38}$ at the Australian Nuclear Science and Technology Organisation (ANSTO). A $2.142 \mathrm{~g}$ sample was loaded into a 6-mm-diameter cylindrical vanadium can inside a helium-filled glovebox and attached to a custom-designed gas-delivery centrestick equipped with in situ gas delivery capabilities, which has been described elsewhere ${ }^{39}$. The incident neutron wavelength $(\sim 2.41 \AA)$ was refined against an $\mathrm{Al}_{2} \mathrm{O}_{3}$ standard reference material (NIST SRM 676). NPD data were collected in the angular range $17^{\circ}<2 \theta<137^{\circ}$ at various temperature intervals upon heating, with the sample temperature stabilised within $1 \mathrm{~K}$ of the set point prior to data acquisition. A Hiden Isochema IMI manometric dosing system was then used to dose the sample cumulatively with aliquots of $\mathrm{CO}_{2}$ gas loaded into a dosing manifold of calibrated volume, allowing the quantity of gas in each dose to be determined from the manifold pressure. All gas doses were applied to the sample at $220 \mathrm{~K}$ followed by slow cooling to $15 \mathrm{~K}$ to avoid the deposition of solid $\mathrm{CO}_{2}$ in the sample chamber; the attainment of near-zero pressure measured inside the sample vessel was taken to indicate complete uptake of the dose by the sample. The entire series of variable-temperature NPD measurements was repeated after each $\mathrm{CO}_{2}$ dose.

Data reduction was performed using LAMP ${ }^{40}$ and included a correction applied for the Debye-Scherrer ring curvature. Lattice parameters for the Co-NP phase were extracted using the Le Bail method as implemented in the program GSAS ${ }^{41}$, which was used in conjunction with the EXPGUI ${ }^{42}$ graphical interface. A pseudo-Voigt peak profile with axial divergence (GSAS Type II) and 16-term shifted Chebyshev background function were fitted to the data. Manual analysis was carried out for the first NPD pattern in each temperature series, followed by sequential (automated) Le Bail extractions over the rest of the series, using the final refined parameters from the previous pattern as a starting point for each subsequent extraction.

Details of the NPD experiments and data treatments for FeCo-PB were very similar to those described here for Co-NP and can be found in Supplementary Information accompanying a previous publication by Ogilvie et al. ${ }^{37}$. Examples of the Le Bail fit profiles obtained for FeCo-PB and Co-NP are shown in Supplementary Fig. 1 accompanying this article, and agreement indices for all fits are summarised in Supplementary Table 1.

\section{Data availability}

The authors declare that the data supporting the findings of this study are available within the article and its Supplementary Information files. All other relevant data supporting the findings of this study are available on request.

Received: 25 May 2018 Accepted: 21 August 2018

Published online: 19 November 2018

\section{References}

1. Cook, T. R., Zheng, Y.-R. \& Stang, P. J. Metal-organic frameworks and selfassembled supramolecular coordination complexes: comparing and contrasting the design, synthesis, and functionality of metal-organic materials. Chem. Rev. 113, 734-777 (2013).

2. Furukawa, H., Cordova, K. E., O'Keeffe, M. \& Yaghi, O. M. The chemistry and applications of metal-organic frameworks. Science 341, 974 (2013).

3. Eddaoudi, M. et al. Systematic design of pore size and functionality in isoreticular MOFs and their application in methane storage. Science 295 469-472 (2002).

4. Eddaoudi, M., Li, H. \& Yaghi, O. M. Highly porous and stable metal-organic frameworks: structure design and sorption properties. J. Am. Chem. Soc. 122, 1391-1397 (2000).

5. McDonald, T. M., D’Alessandro, D. M., Krishna, R. \& Long, J. R. Enhanced carbon dioxide capture upon incorporation of N,N 
'-dimethylethylenediamine in the metal-organic framework CuBTTri. Chem. Sci. 2, 2022-2028 (2011).

6. Sumida, K. et al. Carbon dioxide capture in metal-organic frameworks. Chem. Rev. 112, 724-781 (2012).

7. D'Alessandro, D. M., Smit, B. \& Long, J. R. Carbon dioxide capture: prospects for new materials. Angew. Chem. Int. Ed. 49, 6058-6082 (2010).

8. Li, J.-R., Kuppler, R. J. \& Zhou, H.-C. Selective gas adsorption and separation in metal-organic frameworks. Chem. Soc. Rev. 38, 1477-1504 (2009).

9. Suh, M. P., Park, H. J., Prasad, T. K. \& Lim, D. W. Hydrogen storage in metalorganic frameworks. Chem. Rev. 112, 782-835 (2012).

10. Cairns, A. B. et al. Giant negative linear compressibility in zinc dicyanoaurate. Nat. Mater. 12, 212 (2013).

11. Duyker, S. G., Peterson, V. K., Kearley, G. J., Studer, A. J. \& Kepert, C. J. Extreme compressibility in $\mathrm{LnFe}(\mathrm{CN})_{6}$ coordination framework materials via molecular gears and torsion springs. Nat. Chem. 8, 270-275 (2016).

12. Duyker, S. G., Peterson, V. K., Kearley, G. J., Ramirez-Cuesta, A. J. \& Kepert, C. J. Negative thermal expansion in $\mathrm{LnCo}(\mathrm{CN})_{6}(\mathrm{Ln}=\mathrm{La}, \mathrm{Pr}, \mathrm{Sm}, \mathrm{Ho}, \mathrm{Lu}, \mathrm{Y})$ : mechanisms and compositional trends. Angew. Chem. Int. Ed. 52, 5266-5270 (2013).

13. Chapman, K. W., Chupas, P. J. \& Kepert, C. J. Direct observation of a transverse vibrational mechanism for negative thermal expansion in $\mathrm{Zn}(\mathrm{CN})_{2}$ : an atomic pair distribution function analysis. J. Am. Chem. Soc. 127, 15630-15636 (2005).

14. Goodwin, A. L. \& Kepert, C. J. Negative thermal expansion and low-frequency modes in cyanide-bridged framework materials. Phys. Rev. B 71, 140301 (2005).

15. Dubbeldam, D., Walton, K. S., Ellis, D. E. \& Snurr, R. Q. Exceptional negative thermal expansion in isoreticular metal-organic frameworks. Angew. Chem. Int. Ed. 46, 4496-4499 (2007).

16. $\mathrm{Wu}, \mathrm{Y}$. et al. Negative thermal expansion in the metal-organic framework material $\mathrm{Cu}_{3}(1,3,5 \text {-benzenetricarboxylate })_{2}$. Angew. Chem. Int. Ed. 47, 8929-8932 (2008).

17. Goodwin, A. L., Chapman, K. W. \& Kepert, C. J. Guest-dependent negative thermal expansion in nanoporous Prussian blue analogues $\mathrm{M}^{\mathrm{II}} \mathrm{Pt}^{\mathrm{IV}}(\mathrm{CN})_{6} \cdot \mathrm{x}$ $\left\{\mathrm{H}_{2} \mathrm{O}\right\}(0 \leq \mathrm{x} \leq 2 ; \mathrm{M}=\mathrm{Zn}, \mathrm{Cd})$. J. Am. Chem. Soc. 127, 17980-17981 (2005).

18. Phillips, A. E., Goodwin, A. L., Halder, G. J., Southon, P. D. \& Kepert, C. J. Nanoporosity and exceptional negative thermal expansion in single-network cadmium cyanide. Angew. Chem. Int. Ed. 47, 1396-1399 (2008).

19. Goodwin, A. L., Kennedy, B. J. \& Kepert, C. J. Thermal expansion matching via framework flexibility in zinc dicyanometallates. J. Am. Chem. Soc. 131, 6334-6335 (2009).

20. Chapman, K. W., Southon, P. D., Weeks, C. L. \& Kepert, C. J. Reversible hydrogen gas uptake in nanoporous Prussian blue analogues. Chem. Commun. 3322-3324 (2005).

21. Chapman, K. W., Chupas, P. J. \& Kepert, C. J. Compositional dependence of negative thermal expansion in the Prussian blue analogues $\mathrm{M}^{\mathrm{II}} \mathrm{Pt}^{\mathrm{IV}}(\mathrm{CN})_{6}$ ( $\mathrm{M}=\mathrm{Mn}, \mathrm{Fe}, \mathrm{Co}, \mathrm{Ni}, \mathrm{Cu}, \mathrm{Zn}, \mathrm{Cd})$. J. Am. Chem. Soc. 128, 7009-7014 (2006).

22. Herren, F., Fischer, P., Ludi, A. \& Haelg, W. Neutron diffraction study of Prussian blue, $\mathrm{Fe} 4[\mathrm{Fe}(\mathrm{CN}) 6] 3 . \mathrm{xH} 2 \mathrm{O}$. Location of water molecules and longrange magnetic order. Inorg. Chem. 19, 956-959 (1980).

23. Yang, J., Wang, H., Lu, L., Shi, W. \& Zhang, H. Large-scale synthesis of Berlin Green $\mathrm{Fe}\left[\mathrm{Fe}(\mathrm{CN})_{6}\right]$ microcubic crystals. Cryst. Growth Des. 6, 2438-2440 (2006).

24. Domenech-Carbo, A., Scholz, F. \& Montoya, N. Solvent-independent electrode potentials of solids undergoing insertion electrochemical reactions: Part III. Experimental data for Prussian blue undergoing electron exchange coupled to cation exchange. J. Phys. Chem. C 116, 25993-25999 (2012).

25. Kaye, S. S. \& Long, J. R. Hydrogen storage in the dehydrated Prussian blue analogues $\mathrm{M}_{3}\left[\mathrm{Co}(\mathrm{CN})_{6}\right]_{2}(\mathrm{M}=\mathrm{Mn}, \mathrm{Fe}, \mathrm{Co}, \mathrm{Ni}, \mathrm{Cu}, \mathrm{Zn})$. J. Am. Chem. Soc. 127, 6506-6507 (2005).

26. Gómez, A., Rodríguez-Hernández, J. \& Reguera, E. Crystal structures of cubic nitroprussides: $\mathrm{M}\left[\mathrm{Fe}(\mathrm{CN})_{5} \mathrm{NO}\right] \cdot \mathrm{xH}_{2} \mathrm{O}(\mathrm{M}=\mathrm{Fe}, \mathrm{Co}, \mathrm{Ni})$. Obtaining structural information from the background. Powder Diffr. 22, 27-34 (2007).

27. $\mathrm{Hu}, \mathrm{L}$. et al. Zero thermal expansion and ferromagnetism in cubic $\mathrm{Sc}_{1-\mathrm{x}} \mathrm{M}_{\mathrm{x}} \mathrm{F}_{3}$ $(\mathrm{M}=\mathrm{Ga}, \mathrm{Fe})$ over a wide temperature range. J. Am. Chem. Soc. 136, 13566-13569 (2014)

28. Tallentire, S. E. et al. Systematic and controllable negative, zero, and positive thermal expansion in cubic $\mathrm{Zr}_{1-\mathrm{x}} \mathrm{Sn}_{\mathrm{x}} \mathrm{Mo}_{2} \mathrm{O}_{8}$. J. Am. Chem. Soc. 135, 12849-12856 (2013).

29. Pretsch, T., Chapman, K. W., Halder, G. J. \& Kepert, C. J. Dehydration of the nanoporous coordination framework $\mathrm{Er}^{\mathrm{III}} \mathrm{U}\left[\mathrm{Co}^{\mathrm{III}}(\mathrm{CN})_{6}\right] \cdot 4\left(\mathrm{H}_{2} \mathrm{O}\right)$ : single crystal to single crystal transformation and negative thermal expansion in $\mathrm{Er}^{\mathrm{III}}$ $\left[\mathrm{Co}^{\mathrm{III}}(\mathrm{CN})_{6}\right]$. Chem. Commun. 1857-1859 (2006).

30. Duyker, S. G. et al. Topotactic structural conversion and hydration-dependent thermal expansion in robust $\mathrm{LnM}^{\mathrm{III}}(\mathrm{CN})_{6} \cdot n \mathrm{H}_{2} \mathrm{O}$ and flexible $\mathrm{ALnFe}^{\mathrm{II}}$ $(\mathrm{CN})_{6} \cdot n \mathrm{H}_{2} \mathrm{O}$ frameworks $(\mathrm{A}=\mathrm{Li}, \mathrm{Na}, \mathrm{K} ; \mathrm{Ln}=\mathrm{La}-\mathrm{Lu}, \mathrm{Y} ; \mathrm{M}=\mathrm{Co}$, Fe; $0 \leq n \leq 5)$. Chem. Sci. 5, 3409-3417 (2014).

31. Grobler, I., Smith, V. J., Bhatt, P. M., Herbert, S. A. \& Barbour, L. J. Tunable anisotropic thermal expansion of a porous zinc(II) metal-organic framework. J. Am. Chem. Soc. 135, 6411-6414 (2013).
32. Zhou, H.-L., Zhang, Y.-B., Zhang, J.-P. \& Chen, X.-M. Supramolecular-jacklike guest in ultramicroporous crystal for exceptional thermal expansion behaviour. Nat. Commun. 6, 6917 (2015)

33. Phillips, A. E., Halder, G. J., Chapman, K. W., Goodwin, A. L. \& Kepert, C. J. Zero thermal expansion in a flexible, stable framework: tetramethylammonium copper(I) zinc(II) cyanide. J. Am. Chem. Soc. 132, 10-11 (2010).

34. Gao, Q. et al. Switching between giant positive and negative thermal expansions of a $\mathrm{YFe}(\mathrm{CN})_{6}$-based Prussian blue analogue induced by guest species. Angew. Chem. Int. Ed. 56, 9023-9028 (2017).

35. Wang, X., Hanson, J. C., Szanyi, J. \& Rodriguez, J. A. Interaction of $\mathrm{H}_{2} \mathrm{O}$ and $\mathrm{NO}_{2}$ with $\mathrm{BaY}$ faujasite: complex contraction/expansion behavior of the zeolite unit cell. J. Phys. Chem. B 108, 16613-16616 (2004).

36. Chevreau, $\mathrm{H}$. et al. Concentration-dependent binding of $\mathrm{CO}_{2}$ and $\mathrm{CD}_{4}$ in UiO-66(Zr). J. Phys. Chem. C 119, 6980-6987 (2015).

37. Ogilvie, S. H., Duyker, S. G., Southon, P. D., Peterson, V. K. \& Kepert, C. J. Identification of bridged $\mathrm{CO}_{2}$ binding in a Prussian blue analogue using neutron powder diffraction. Chem. Commun. 49, 9404-9406 (2013).

38. Studer, A. J., Hagen, M. E. \& Noakes, T. J. Wombat: the high-intensity powder diffractometer at the OPAL reactor. Phys. B: Condens. Matter 385-386, 1013-1015 (2006).

39. Lee, S. et al. Powder sample-positioning system for neutron scattering allowing gas delivery in top-loading cryofurnaces. J. Appl. Crystallogr. 49, 705-711 (2016).

40. LAMP, the Large Array Manipulation Program, Institut Laue-Langevin (1996)

41. Larson, A. C. \& Von Dreele, R. B. General Structure Analysis System (GSAS). Los Alamos National Laboratory Report LAUR, NIST, Gaithersburg, USA, 86748. (1994).

42. Toby, B. H. EXPGUI, a graphical user interface for GSAS. J. Appl. Crystallogr. 34, 210-213 (2001).

\section{Acknowledgements}

This work was supported by the Science and Industry Endowment Fund (RP02-035). S. H.O. acknowledges the Australian Institute of Nuclear Science and Engineering for a postgraduate research award. S.G.D. thanks the University of Sydney for fellowship funding. C.J.K. thanks the Australian Research Council for Discovery Project (DP150104620) and Fellowship (FT100100514) funding. The authors thank the sample environment team at the Australian Centre for Neutron Scattering for assistance with the gas delivery system used during the NPD experiments.

\section{Author contributions}

J.E.A. developed the multisite empirical data model and drafted the manuscript. A.A.B and S.H.O. synthesised the materials, analysed the NPD data and developed the initial data model. S.G.D., H.C. and V.K.P. performed the NPD/gas delivery experiments. S.G. D., V.K.P. and C.J.K. directed and guided the research project. All authors participated in discussions and revision of the manuscript.

\section{Additional information}

Supplementary Information accompanies this paper at https://doi.org/10.1038/s41467018-06850-6.

Competing interests: The authors declare no competing interests.

Reprints and permission information is available online at http://npg.nature.com/ reprintsandpermissions/

Publisher's note: Springer Nature remains neutral with regard to jurisdictional claims in published maps and institutional affiliations.

Open Access This article is licensed under a Creative Commons Attribution 4.0 International License, which permits use, sharing, adaptation, distribution and reproduction in any medium or format, as long as you give appropriate credit to the original author(s) and the source, provide a link to the Creative Commons license, and indicate if changes were made. The images or other third party material in this article are included in the article's Creative Commons license, unless indicated otherwise in a credit line to the material. If material is not included in the article's Creative Commons license and your intended use is not permitted by statutory regulation or exceeds the permitted use, you will need to obtain permission directly from the copyright holder. To view a copy of this license, visit http://creativecommons.org/licenses/by/4.0/.

(C) The Author(s) 2018 